\title{
Archives
}

\section{De la théorie à la pratique : l'exemple des stages de la Division de la gestion de documents et des archives de l'Université de Montréal}

\section{ISABELLE DION}

Volume 46, numéro 2, 2017

$50^{\mathrm{e}}$ anniversaire de la DGDA

URI : https://id.erudit.org/iderudit/1040382ar

DOI : https://doi.org/10.7202/1040382ar

Aller au sommaire du numéro

Éditeur(s)

Association des archivistes du Québec (AAQ)

ISSN

0044-9423 (imprimé)

2369-9256 (numérique)

Découvrir la revue

Citer cet article

Dion, I. (2017). De la théorie à la pratique : l'exemple des stages de la Division de la gestion de documents et des archives de l'Université de Montréal. Archives, 46(2), 91-112. https://doi.org/10.7202/1040382ar
Résumé de l'article

Le stage est une composante majeure de la formation pratique dans un programme d'études qui permet à l'étudiant d'acquérir, entre autres, de nouvelles connaissances, de développer ses compétences et de réfléchir sur son comportement en situation réelle. Depuis leur création, les programmes d'enseignement en archivistique offrent des stages pratiques dans des services de gestion de documents et des archives. L'auteure met en lumière l'expérience des stages à la Division de la gestion de documents et des archives de l’Université de Montréal qui reçoit depuis ses débuts des stagiaires en archivistique de tous les niveaux d'enseignement, d'ici et d'ailleurs. L'article reflète le point de vue de la coordonnatrice de stages et explique les nombreux apports de ces stages pour les étudiants en archivistique. 


\section{ANNIVERSAIRE DE LA DGDA}

\section{De la théorie à la pratique: l'exemple des stages de la Division de la gestion de documents et des archives de I'Université de Montréal}


les ressemblances et différences entre la théorie et la pratique. Depuis leur création, les programmes d'enseignement en archivistique offrent des stages pratiques dans des services de gestion de documents et des archives. Ces services accueillent avec enthousiasme des étudiants curieux et passionnés par leur future profession.

Dans le cadre des cours, les travaux pratiques, les études de cas, les activités dirigées, les visites dans les milieux, les exercices en laboratoire, les interventions dans certains milieux et les bilans d'expérience par des conférenciers préparent l'étudiant à une certaine pratique des fonctions archivistiques. Le stage, contrairement aux cours, se déroule dans un milieu de travail où les priorités, les tâches et les méthodes changent au fil des situations et innovations, et ne peut être organisé comme dans les cours. L'étudiant a la responsabilité de saisir cette différence et de s'impliquer dans son milieu de stage tant par ses compétences professionnelles que relationnelles.

Le stage marque généralement la fin des études. Dans le cas des programmes en archivistique de l'École de bibliothéconomie et des sciences de l'information (EBSI), il constitue la dernière étape du parcours des étudiants. II représente le passage du statut d'étudiant au statut $d^{\prime}$ archiviste professionnel. Ce passage où l'étudiant devient stagiaire lui procure une expérience en situation de travail et joue un grand rôle dans sa professionnalisation.

Dans le cadre de ce texte, nous souhaitons mettre en lumière l'expérience des stages à la Division de la gestion de documents et des archives (DGDA). Depuis ses débuts, la DGDA reçoit régulièrement des stagiaires en archivistique de tous les niveaux d'enseignement, d'ici et d'ailleurs. Nos réflexions s'attarderont principalement aux stages du certificat en archivistique et de la maîtrise en sciences de l'information de l'EBSI de I'Université de Montréal. Nous présenterons également les stages de la DGDA du point de vue de la coordonnatrice de stages et mettrons de l'avant les nombreux apports de ces stages pour les étudiants en archivistique'.

\section{L'ÉCOLE DE BIBLIOTHÉCONOMIE ET DES SCIENCES DE L'INFORMATION}

L'École de bibliothéconomie et des sciences de l'information est une école professionnelle qui forme des spécialistes en sciences de 
I'information dans quatre programmes distincts: certificat en archivistique, certificat en gestion de l'information numérique, maîtrise en sciences de I'information et doctorat en sciences de l'information.

Un stage de fin d'études non rémunéré est offert aux étudiants inscrits aux programmes du certificat en archivistique et de la maîtrise en sciences de l'information - orientation professionnelle. Le stage au certificat en archivistique est une activité optionnelle du programme. Les étudiants doivent avoir maintenu une moyenne de $B$ et complété la majorité de leurs cours avant d'obtenir l'autorisation de s'y inscrire. Le stage est de 25 jours ouvrables et se réalise au rythme minimum de trois jours par semaine.

L'orientation professionnelle de la maîtrise en sciences de l'information comprend un stage obligatoire de 36 jours qui a pour but de permettre I'approfondissement des connaissances acquises lors du parcours académique (EBSI, 2015, p. 5). Pour s'y inscrire, l'étudiant doit avoir terminé et réussi ses six cours de base obligatoires ainsi que sept des neuf cours à option.

Les évaluations de stage du certificat et de la maîtrise comprennent un journal de bord, une rencontre de groupe de stagiaires qui a lieu à I'EBSI, un rapport de stage et un formulaire d'évaluation des compétences professionnelles et des qualités personnelles du stagiaire, complété par le superviseur du milieu de stage.

Le milieu de stage doit remplir quelques conditions pour recevoir un stagiaire du certificat ou de la maîtrise. À noter qu'il n'y a pas de restriction concernant la taille ou le domaine d'activité des organismes qui peuvent recevoir des stagiaires en archivistique. Tout d'abord, le stagiaire doit être supervisé par un professionnel de la gestion de I'information (archiviste, gestionnaire de documents, etc.) qui sera en mesure d'évaluer la qualité de son travail et de le guider au besoin. Le stagiaire est en période d'apprentissage et ne doit pas jouer le rôle d'un consultant qui apporterait une expertise dont le milieu ne dispose pas. Ensuite, les tâches du stagiaire doivent être de nature professionnelle². L'étudiant peut exécuter des tâches reliées à une ou plusieurs fonctions archivistiques courantes ou travailler à la réalisation d'un projet ponctuel, ou encore combiner les deux types d'activités. Pour finir, le milieu de stage doit offrir des conditions de travail adéquates et fournir les outils de travail nécessaires à la réalisation des activités de stage (par exemple: logiciels, sources d'information) (EBSI, 2015, p. 8). 
Tout comme les cours, les stages ont des objectifs généraux qui doivent être atteints par l'étudiant. Le stage doit permettre à l'étudiant de:

- relier ses connaissances théoriques à une expérience pratique;

- développer des habiletés professionnelles;

- développer des qualités personnelles;

- se situer professionnellement;

- avoir un aperçu de différents milieux de travail.

Ces objectifs s'appliquent autant aux étudiants du certificat en archivistique qu'à tous les étudiants de la maîtrise, peu importe le domaine du stage (archivistique, bibliothéconomie, architecture de l'information, etc.). Les stagiaires réfléchissent à ces objectifs par le biais de leur journal de bord (certificat) ou de leur rapport de stage (maîtrise). Le savoir, le savoir-faire et le savoir-être sont représentés par les trois premiers objectifs. Se situer professionnellement invite l'étudiant à se demander s'il a fait le bon choix professionnel, s'il préfère certaines tâches ou certains milieux de travail. La rencontre de groupe de stagiaires où les stagiaires présentent leur milieu de stage et écoutent leurs collègues faire de même, répond au dernier objectif du stage. À cette occasion, le stagiaire apprend sur d'autres milieux, qui seront éventuellement des milieux de travail, et sur des activités similaires ou différentes des siennes.

\subsection{Quelques réflexions sur les stages ${ }^{3}$}

Avant leur arrivée en stage, les coordonnatrices de stages présentent aux étudiants ce que nous appelons de façon pragmatique la philosophie des stages. Une partie de cet exposé est consacré aux savoirs, savoir-faire et savoir-être appliqués en classe, mais tout aussi importants en stage. En stage, l'étudiant apprend encore. II poursuit ses lectures (politiques et procédures, rapport annuel, site Web, etc.) afin de connaître son milieu de stage et surtout il observe ce qui se passe autour de lui. II remarque les tâches effectuées par son superviseur et ses interactions avec ses collègues de travail. L'étudiant observe aussi le travail du personnel du service d'archives et la clientèle (usagers internes et externes). II apprend comment agir lors de certaines situations (ex.: comment demander des précisions à un usager, comment demander de l'aide à un membre du personnel pour réaliser une tâche, etc.). 
L'étudiant développe également son savoir-faire en stage. Ses activités de stage lui permettent d'améliorer ses compétences et habiletés. Il connaît la théorie, maintenant c'est le moment de faire des tâches: traiter un fonds d'archives, concevoir et appliquer un plan de classification ou des règles de conservation, sélectionner des documents d'archives pour une exposition, appliquer une politique de nommage, etc. II utilise les outils archivistiques de son milieu de stage et découvre des méthodes de travail, par exemple comment s'organiser physiquement pour traiter un fonds d'archives ou comment réaliser efficacement et rapidement un inventaire de documents numériques.

Enfin, l'étudiant aura à mettre de l'avant son savoir-être. Cela est particulièrement important en stage, car tous les stagiaires ont reçu la formation pour exercer la profession d'archiviste, toutefois le comportement et l'attitude en stage d'un stagiaire sont uniques. Le stagiaire doit en prendre conscience, mesurer ses forces et trouver des moyens pour améliorer ses points faibles. Dans un contexte de travail, le stagiaire ne doit pas uniquement se concentrer sur les tâches archivistiques à accomplir, il doit s'intégrer et participer à la vie du service $d^{\prime}$ archives. Cela signifie, outre de faire preuve de professionnalisme dans ses activités, de participer aux discussions lors des pauses et des repas, de développer ses opinions et de savoir les présenter, de comprendre le code vestimentaire de l'organisme, de faire preuve de civisme (ex. : cogner avant d'entrer dans un bureau, saluer les personnes rencontrées) et de souplesse dans le travail (ex. : changement à l'horaire ou aux activités). Le stage est un bon moment pour réfléchir sur son comportement. Avant son entrée sur le marché du travail, le stagiaire reconnaîtra ses compétences, ses forces et ses faiblesses. II sera d'autant mieux préparé aux entrevues qui suivront la fin de ses études.

Le stagiaire est aussi responsable de sa formation lors du stage. II est de sa responsabilité d'acquérir de nouveaux apprentissages. En classe, I'enseignant peut insister sur certains éléments théoriques et demander à l'étudiant de réaliser des travaux pratiques ou de recherche. En stage, c'est à lui de transformer ses observations, ses activités, son comportement en occasion d'apprentissage.

II applique les connaissances acquises, en découvre d'autres, s'implique, s'intègre, pose des gestes, réalise des activités, résout des problèmes. $\|$ doit s'assurer de mener à bien son 
projet de stage. Son rôle est aussi de prendre en main sa formation, de développer les mécanismes pour apprendre par lui-même, pour se questionner sur ce qu'il sait, sur ce qu'il voit et sur ce qu'il pense.

Ainsi, si l'étudiant exploite son expérience de stage et qu'il réfléchit quotidiennement sur les activités et sur l'ensemble de son expérience, il bonifie son apprentissage théorique, acquiert de nouvelles connaissances et atteint véritablement les objectifs de stage (Arès, 1989, p. 59).

Pour créer des situations d'apprentissage, l'étudiant doit se montrer intéressé. Intéressé par ses tâches, celles des autres, se renseigner sur son milieu de stage avant et pendant le stage. Poser des questions facilite l'appropriation de nouveaux apprentissages. Évidemment, le stagiaire doit réfléchir avant de poser des questions. II doit formuler des hypothèses de réponses qui peuvent alimenter la discussion avec son superviseur ou les membres de l'équipe du service d'archives. II doit être préparé, car son superviseur ou toute autre personne pourrait bien lui retourner la question en lui demandant ce qu'il en pense! Enfin, se montrer ouvert à des aspects différents de la profession amène de nouveaux apprentissages pour le stagiaire. Que ce soit une méthode utilisée différente de celle apprise en classe, un conseil pour accélérer ou organiser une activité ou une décision qui semble surprenante, le stagiaire profitera du fait d'être ouvert à la nouveauté sous toutes ses formes. La pratique dans un milieu archivistique est inévitablement différente de la théorie enseignée. Les questions (ex.: pourquoi faites-vous les choses de cette façon?) et les réflexions (retour à la théorie, compréhension du contexte) du stagiaire viendront alimenter ses apprentissages.

\section{LES STAGES À LA DIVISION DE LA GESTION DE DOCUMENTS ET DES ARCHIVES}

L'EBSI et la DGDA entretiennent des liens archivistiques depuis fort longtemps. En plus d'être un milieu de stage, la DGDA participe activement à des travaux pratiques pour certains cours (exercice d'application de règles de conservation, activités de diffusion). Plusieurs membres de son équipe ont enseigné comme chargés de cours au certificat et à la maîtrise, et bon nombre d'entre eux viennent présenter un aspect particulier de la 
Division ou de la profession à titre de conférencier dans le cadre des cours. Michel Champagne a déjà bien documenté les stages à la DGDA dans son article Le rôle stratégique du stage dans la formation en archivistique: réflexions d'un superviseur de stage (2010-2011). Aujourd'hui, ce sont plutôt les étapes des stages de la DGDA qui seront abordées du point de vue d'une coordonnatrice de stages.

\subsection{Processus de placement}

Le processus de placement des stagiaires débute avec une rencontre individuelle entre la coordonnatrice de stages et chaque étudiant désirant réaliser son stage au trimestre suivant. Le but de cette rencontre est de connaître le parcours de l'étudiant, de discuter du choix de l'archivistique comme domaine d'études et de profession ainsi que de ses préférences en matière d'activités de stage. Généralement, les stages comprennent des activités en lien avec les archives historiques ou en lien avec la gestion des documents actifs et semi-actifs. Parfois, un étudiant propose une activité particulière qui lui tient à cœur, car elle est en lien avec ses études ou expériences antérieures (par exemple, travailler avec des documents audiovisuels en raison d'un baccalauréat en cinéma). Une discussion a lieu également avec le milieu de stage pour connaître ses besoins et ses priorités.

Dans le cas de la DGDA, les projets de stage tiennent compte de l'intérêt de l'étudiant pour la gestion documentaire ou les archives historiques, mais également d'autres intérêts qui pourraient se présenter (intérêt pour les sciences de la nature ou la musique, intérêt à concevoir un texte pour les réseaux sociaux, etc.). Par la suite, la coordonnatrice de stages suggère un étudiant à l'un des archivistes de la Division selon le profil et les intérêts de celui-ci et envoie son curriculum vitæ. Pour les étudiants du certificat en archivistique, I'archiviste-superviseur peut demander à rencontrer l'étudiant pour discuter d'activités précises. De leur côté, les étudiants de la maîtrise doivent prendre rendez-vous avec l'archiviste-superviseur pour discuter des activités de stage et les inscrire sur le formulaire d'entente de stage.

Michel Champagne énumère ses attentes envers les stagiaires qu'il accueille (2010-2011, p. 112). La première est que l'étudiant doit avoir une bonne formation de base. Le stage amène l'application des connaissances et non l'acquisition de celles-ci. Ensuite, «[...] les stagiaires doivent être curieux et intéressés par leur milieu de stage et par le travail d'archiviste » 
(Champagne, 2010-2011, p. 112). La motivation d'un stagiaire se voit rapidement par les discussions, les questions posées, son ouverture d'esprit, son désir de réaliser un travail bien fait en le validant auprès de son superviseur. Enfin, les stagiaires doivent être autonomes. Au début de la période de stage, il y a davantage de questions, de validations. Ces dernières diminuent avec le temps, car le stagiaire sait ce qu'il a à faire et comment le faire. L'autonomie, c'est aussi de se questionner avant de poser une question à son superviseur, de rassembler les questions ou problèmes non résolus pour ensuite rencontrer son superviseur à un moment opportun.

\subsection{Le profil des stagiaires}

Un mot définit très bien le profil des stagiaires de la DGDA: diversifié. Faisant preuve d'une grande ouverture, la Division accueille des stagiaires de tous les âges, qui arrivent directement du collégial, qui ont un baccalauréat et parfois même une maîtrise, qui font un retour aux études après quelques années ou une longue période ou encore qui amorcent un changement de carrière. Ainsi, de très jeunes étudiants $d$ 'à peine vingt ans jusqu'à ceux d'une cinquantaine d'années se sont succédé à la Division. Beaucoup d'étudiants d'origine québécoise y ont fait leur stage, mais aussi des étudiants provenant de tous les continents. Quelques étudiants en situation de handicap ont également bénéficié d'un stage adapté à leurs besoins.

La très grande majorité des stagiaires n'avait aucune expérience préalable en archivistique avant de réaliser leur stage. Quelques étudiants avaient un peu d'expérience à la suite d'un travail d'été étudiant. Certains étudiants de la maîtrise ont suivi antérieurement le certificat en archivistique incluant un stage. Quelques étudiants en programme d'échange en provenance de I'Université de Mulhouse et de l'École des Chartes en France ont accompli un stage dans le programme de leur université d'attache avant de procéder à leur stage à la DGDA dans le cadre de la maîtrise en sciences de l'information.

\subsection{Les projets de stage}

Le plan de stage type de la DGDA comporte un certain nombre d'activités récurrentes, peu importe s'il s'agit d'un stage au certificat ou à la maîtrise. 
Tout d'abord, la première journée est consacrée à l'intégration du stagiaire à la Division par la présentation des membres de l'équipe, la culture organisationnelle (dîner, pauses, code vestimentaire, etc.) et la visite des bureaux. La visite des dépôts de documents semi-actifs et inactifs se déroule au cours du stage selon l'avancement des activités. La connaissance de la DGDA se poursuit avec la lecture du rapport annuel et des procédures en vigueur. L'étudiant visionne par la suite des vidéos présentant les activités de la DGDA. L'étudiant peut aussi naviguer sur le site Web de la Division et parcourir toutes ses sections. Ces informations lui seront utiles non seulement comme apprentissage du milieu, mais également lorsqu'il aura à présenter son milieu de stage à ses collègues et lors de la rédaction de son rapport de stage. Enfin, I'archiviste-superviseur discute avec le stagiaire des activités qu'il aura à accomplir au cours des prochains jours.

La DGDA a mis en place une formule de stage qui permet à l'étudiant de comprendre l'ensemble des tâches archivistiques effectuées dans son service même si ce dernier ne réalisera pas toutes ces tâches. Ainsi, si l'étudiant a un stage misant davantage sur la gestion des archives historiques, il aura quand même accès à des informations importantes sur la gestion des documents actifs et semi-actifs par l'entremise de rencontres avec le personnel de la Division. Le stage comprend des rencontres avec les techniciens et les professionnels qui s'échelonnent tout au long du stage. Chaque rencontre s'étend sur quelques heures ou une demi-journée. Toutes les fonctions de l'archivistique sont mises en pratique à la DGDA. Par conséquent, l'étudiant a une vision globale et intégrée de l'archivistique.

Les rencontres avec le personnel portent sur l'acquisition des archives privées, le traitement préliminaire et définitif des archives ${ }^{4}$, la présentation de la base de données (SyGED), le calendrier de conservation et son application, la gestion des semi-actifs, le traitement dans les unités administratives, le système de classification, les transferts des documents, la formation des usagers, la gestion et l'analyse des procès-verbaux, l'analyse des contrats, la numérisation, la référence, la présentation du site Web et la gestion d'un service d'archives. Cela exige une importante logistique puisque plusieurs employés sont impliqués et doivent dégager de leur temps pour en consacrer au stagiaire. Ce choix a cependant un impact très positif sur le stagiaire. En plus d'accélérer son intégration dans le milieu en rencontrant le personnel de la Division, cette addition aux activités de stage permet à l'étudiant de comprendre toutes les tâches 
archivistiques, de faire des liens entre elles, de poser des questions en lien avec la théorie, de combler ses «trous» dans la chaîne archivistique.

Les stages de la DGDA des étudiants du certificat en archivistique comprennent plusieurs activités. Le stagiaire qui souhaite un stage en gestion des documents actifs et semi-actifs participera à la réception des transferts des documents en provenance des unités administratives, l'application de règles de conservation, l'élimination de documents, le transfert de documents numériques dans le logiciel de gestion documentaire, l'indexation de procès-verbaux ou de contrats, aux rappels, retours et ajouts de documents. II pourra aussi numériser des documents pendant quelques heures ou quelques jours afin de se familiariser avec ce processus. Du côté de la gestion des archives historiques, le stagiaire prend part au traitement préliminaire de fonds d'archives, au traitement des archives imprimées, à l'indexation de pièces ou de dossiers, à la mise à jour de l'état général des fonds, à la numérisation de documents et au traitement des images, au versement de documents, à la description de documents iconographiques, au traitement et au transfert d'archives numériques. Si l'occasion se présente, il collabore à des activités de diffusion telles que des expositions virtuelles ou la création de contenus pour les médias sociaux. II peut également faire le traitement définitif d'un fonds d'archives (inventaire, classification, classement, description, indexation, préservation $)^{5}$.

Au cours des dernières années, des stagiaires du certificat ont contribué à des projets particuliers: I'analyse en vue de la numérisation des données des règles de conservation (2009), la classification des documents numériques en vue de leur dépôt dans un système de GID (2008), I'implantation de la gestion documentaire au Bureau des communications et des relations publiques et à la Faculté des sciences infirmières (2012), la participation à la normalisation et le paramétrage de SyGED (2012), la rédaction d'un rapport sur la transposition de différentes classifications personnalisées au système officiel de classification (2012).

Du côté de la maîtrise en sciences de l'information, les projets de stage peuvent inclure des activités régulières de la Division. II peut s'agir de participer à des projets d'implantation en cours, d'appliquer le système de classification, de s'initier à la numérisation, de normaliser l'indexation, de contribuer au contenu des dossiers de personnalités ou des médias sociaux, de collaborer à un projet de diffusion, de mettre à jour l'état 
général des fonds et le site Web, de faire le versement de documents, de décrire et indexer des documents à la pièce. Certains étudiants de la maîtrise font le traitement définitif de fonds d'archives ${ }^{6}$. En plus de certaines de ces activités, le stagiaire a son propre projet à mener. Ce projet est la plupart du temps la combinaison entre les souhaits des stagiaires et les priorités de la Division. Voici une liste non exhaustive de ces projets:

- étude sur les documents informatiques (1992);

- méthode d'inventaire des équipements de rangement (1992);

- étude et tests de classification (1994);

- aide à la conception du site Web (1999);

- analyse des procès-verbaux et des avis de nomination ou de création ou modification d'un organisme; élaboration de méthodes d'analyse (1999);

- réalisation de l'exposition virtuelle Sciences \& modernité, la petite faculté de luxe de 1920 à 1945 (2000);

- révision des procédures d'indexation et la mise à jour des descripteurs (2000 et 2001);

- participation à la section Documents numérisés du Service de la gestion de documents et des archives (2002);

- révision du calendrier spécifique des règles de conservation de la Faculté de l'éducation permanente (2005);

- étude sur les documents audiovisuels (2005);

- participation à la rédaction du Guide de classification (2006);

- étude de faisabilité du transfert de bandes vidéo conservées par le Service de la gestion de documents et des archives (2009);

- réalisation de l'exposition virtuelle Hans Selye (2010);

- révision et rédaction de procédures relatives à la gestion des documents semi-actifs (2011);

- rédaction d'un cahier des charge pour la numérisation sérielle des documents au sein des universités du Québec en collaboration 
avec le Service de gestion de documents et des archives de HEC Montréal (2011);

- conception de capsules de formation en ligne à l'attention du personnel de I'Université (2013);

- guide de procédures pour la numérisation des factures liées à des bons de commande de la Direction des finances (2013);

- rédaction d'un projet de politique sur les courriels (2014);

- projet de catégorisation des actifs informationnels des dossiers des membres du personnel (2014);

- réalisation d'une formation en ligne sur les documents essentiels (2015).

Ces exemples de projets de stage amènent les étudiants à contribuer aux préoccupations actuelles de la Division, à analyser une situation et à proposer des recommandations. Dans plusieurs cas, les étudiants doivent contacter d'autres membres du personnel de l'Université et même des professionnels à l'extérieur de celle-ci. Ils font de la gestion de projet à petite échelle, toutefois cela leur donne une bonne idée de la réalité et des difficultés qui peuvent survenir en cours de route. Ils deviennent des professionnels en action.

\subsection{L'encadrement}

Le milieu de stage, tout en bénéficiant du travail du stagiaire, participe à la formation de futurs professionnels de l'information. Il doit offrir une supervision régulière afin de s'assurer que le stagiaire comprend bien les activités à faire. Selon le projet de stage, un ou deux superviseurs sont désignés pour accompagner le stagiaire. Le superviseur répond aux questions de l'étudiant, vérifie la qualité de son travail et discute des problèmes rencontrés.

Le superviseur planifie les activités de stage. II accueille le stagiaire et l'initie à la réalité de son milieu. Lors de l'accueil, le superviseur clarifie le mandat du stagiaire en précisant les attentes. II fournit au stagiaire toute l'information nécessaire au bon déroulement du stage. II I'accompagne dans ses démarches d'apprentissage. Son rôle est de bien 
expliquer ce qu'il faut faire et comment le faire si besoin est; mais il devient pédagogue surtout lorsqu'il prend le temps de préciser pourquoi il faut le faire de cette façon. (Arès, 1989, p. 58)

Les superviseurs de stage de la DGDA établissent à l'avance les activités de stage de l'étudiant. Ces activités sont diversifiées et permettent le développement des compétences professionnelles des étudiants. Comme le mentionne Michel Champagne, «[i]l faut établir un bon dosage d'activités pour que le stage soit varié sans être superficiel » (2010-2011, p. 110). Au formulaire d'entente de stage dans lequel sont énumérées les activités de stage s'ajoute parfois un horaire détaillé du travail qui sera fait chaque jour.

Le superviseur de stage de la Division est toujours disponible pour répondre aux questions du stagiaire. Si ce n'est pas immédiatement, il pourra répondre au stagiaire au cours de la journée. Cette disponibilité établit rapidement un lien de confiance entre le superviseur et le stagiaire. Villeneuve mentionne qu'au début de son stage, «[...] la vulnérabilité du stagiaire est très grande. Son inexpérience et son incapacité temporaire le mettent en position d'insécurité et de dépendance» $(1995$, p. 2). Ces moments de rencontre entre le superviseur et l'étudiant permettent de faire le point régulièrement sur le déroulement du stage et de procéder à des modifications au besoin. À la Division, il a au moins une rencontre hebdomadaire pour faire le point sur l'avancement des activités et répondre aux interrogations du stagiaire. Ces questions peuvent se transformer aussi en discussions, très importantes dans l'apprentissage du milieu et des façons de faire pour l'étudiant.

Plus le stage avance, plus le superviseur connaît le stagiaire et est conscient de ses limites ou de ses progrès. Le superviseur de la DGDA est à l'écoute de son stagiaire. II n'est pas avare de conseils et partage fréquemment ses expériences avec le stagiaire. II est là pour expliquer et dédramatiser les erreurs que le stagiaire peut faire. Ce dernier est encore en période d'apprentissage, alors des erreurs lors de l'accomplissement des tâches peuvent survenir. L'objectif pour le superviseur est de bien démontrer au stagiaire l'origine de l'erreur et les conséquences de celle-ci afin que le stagiaire ne la répète pas et reste motivé. Le commentaire d'une stagiaire de la maîtrise résume bien le suivi effectué par sa superviseure: "[elle] m'a aidée à effectuer un travail professionnel 
grâce à sa disponibilité, ses critiques constructives, son encadrement et son expertise dans le domaine archivistique ${ }^{7}$. »

La productivité est un élément qui inquiète souvent les stagiaires. Ils souhaitent performer comme des professionnels dès leur première expérience en archivistique. Michel Champagne mentionne qu'il « insiste plus sur la qualité du travail à réaliser que sur la quantité de travail » (2010-2011, p. 111). Il faut rappeler à l'étudiant qu'il perdra du temps et donc de la productivité s'il doit recommencer une tâche réalisée avec des erreurs ou omissions.

Le superviseur prend le temps d'évaluer oralement le stagiaire en cours de stage. Cette évaluation permet au stagiaire de se réajuster au besoin. Comme il joue le rôle de conseiller auprès du stagiaire, son but est de participer à l'amélioration des compétences professionnelles et personnelles de l'étudiant. Si l'étudiant connaît ses points à améliorer, il pourra corriger le tir d'ici la fin de son stage. Ce n'est pas seulement le moment de parler des points négatifs, mais aussi de dire au stagiaire les points positifs lors de l'exécution de ses tâches et au sujet de son attitude en stage. À la fin du stage, le superviseur remplit le formulaire d'évaluation de l'étudiant et le lui remet avec ses commentaires.

Les membres du personnel de la DGDA qui acceptent de superviser un étudiant lors de son stage ne minimisent pas l'investissement que demande l'encadrement de ce dernier. Préparer l'arrivée du stagiaire, prévoir pour lui des activités intéressantes, offrir un encadrement adapté à la personnalité ou aux capacités de l'étudiant, allouer du temps pour échanger avec le stagiaire et l'évaluer objectivement, toutes ces responsabilités sont acceptées avec sérieux et enthousiasme par les techniciens et archivistes de la Division.

\section{APPORTS DU STAGE DE LA DGDA POUR L'ÉTUDIANT}

Pour l'étudiant, les apports de son stage réalisé, particulièrement à la DGDA, rejoignent les objectifs généraux des stages dans tous les programmes d'études. Nous développerons ici les apports les plus importants pour les étudiants. Nous avons également écrit aux stagiaires des dernières années afin d'obtenir leurs commentaires sur leur stage à la DGDA. 


\subsection{S'intégrer à un milieu de travail}

Dès les premiers jours de son stage, le stagiaire apprend à s'installer dans un nouveau milieu. II doit se présenter et aller au-devant des gens afin de s'intégrer au personnel du service. Évidemment, l'accueil préparé par le milieu est important. Une stagiaire a souligné qu'elle a «[...] eu un accueil chaleureux de la part d'une équipe dynamique ${ }^{8} »$. L'intégration passe aussi par la compréhension de la culture organisationnelle de I'organisme, non seulement les habitudes du milieu (dîner, pauses, code vestimentaire, etc.), mais également des liens hiérarchiques entre les employés de l'équipe et le travail de chacun. Les présentations faites par chacun des employés de la DGDA aux stagiaires amènent les étudiants à comprendre la nature du travail de chacun ainsi que leur rôle au sein de l'équipe. Pour plusieurs stagiaires, il s'agit de la première expérience en archivistique. Ils découvrent un mode différent de l'environnement scolaire. "Le domaine des archives était nouveau pour moi, alors il s'agissait d'un premier contact avec la vie de chaque jour du monde archivistique $^{9}$. »

\subsection{Faire l'inventaire de ses connaissances et de ses compétences}

Les cours en classe demeurent sensiblement théoriques pour les étudiants. À la DGDA,

[l]e stage contenait également un volet «théorique » très intéressant. Effectivement, nous avions des rencontres planifiées, entre autres, avec la responsable du développement du logiciel de GED et I'archiviste responsable de la gestion des documents dans les différentes unités de l'Université qui nous ont parlé de leurs tâches au quotidien ${ }^{10}$.

Ces rencontres confirment la compréhension de la théorie enseignée et laissent la chance aux stagiaires de poser des questions qu'ils n'auraient pas osé poser en classe ou d'aller plus loin sur certains aspects du travail.

Le stage est aussi «une mise en pratique de connaissances restées jusque-là purement théoriques ${ }^{11} \gg$. Les cours sont généralement présentés selon les fonctions archivistiques et le stage permet aux étudiants de remettre les notions en place dans leur tête, de constater que plusieurs 
fonctions archivistiques s'effectuent en même temps. À ce moment, tout ce qu'ils ont appris pendant leurs études prend une forme concrète. Ils ont quitté le côté obscur pour la lumière! Un stagiaire mentionne à juste titre:

Le stage a été également l'occasion d'assister à de courtes séances d'information pour parfaire dans la réalité quotidienne des notions inculquées sur les bancs d'école. Car de la théorie à la pratique, il se tisse parfois des mondes antagoniques où les écrits peinent à trouver des assises dans le monde concret et la pratique courante de la discipline. J'ai senti au contraire que mes collègues, et une autre stagiaire qui était là en même temps que moi, avaient le souci d'arrimer les dimensions multiples du travail archivistique $^{12}$.

La DGDA est I'un des milieux où la théorie est la plus proche de la pratique. II y est facile de comprendre et d'accepter les écarts entre la théorie et la pratique. Les étudiants s'aperçoivent aussi que le terrain est une réalité autrement plus complexe qu'il n'y paraît au premier coup d'œil. Le simple fait d'observer en stage est une riche source de confirmation théorique et d'apprentissages variés.

En plus d'actualiser ses savoirs, car le milieu de stage évolue constamment, le stagiaire apprend les aspects techniques de la profession. Remplir un bordereau, modifier l'état général des fonds sur le site Web, inscrire les données dans le logiciel sont des exemples de tâches qui n'ont pas nécessairement été vues en détail dans les cours, mais qu'il faut maîtriser en tant que technicien ou archiviste. Le stagiaire doit s'entraîner à la tâche, en assimiler les détails et devenir de plus en plus rapide avec le temps. II devient opérationnel.

Avec une diversité d'activités lors de son stage, l'étudiant peut aller chercher un maximum de connaissances et de compétences archivistiques. En plus des activités qu'il a déjà accomplies lors des cours, il apprend de nouvelles façons de travailler, I'utilisation de nouveaux outils et découvre les tâches dans lesquelles il excelle et celles qu'il doit améliorer. Lors de son stage à la Division, une étudiante mentionne: «[...] j'ai confirmé l'importance d'être méthodique dans la prise d'information: cela peut parfois prendre plus de temps au départ, mais permet d'en gagner bien plus par la suite $!^{13}{ }^{\prime}$ À la fin de son stage, le stagiaire peut faire l'inventaire 
des connaissances acquises et des compétences développées. Il constate aussi son évolution depuis le premier jour de stage.

\subsection{Se développer sur le plan personnel}

En stage, les qualités personnelles sont tout aussi importantes que les compétences professionnelles. Elles font partie intégrante de la réflexion que les stagiaires doivent développer dans leur journal de bord et dans leur rapport de stage, et constituent des critères d'évaluation pour le milieu de stage. Tenue de se questionner sur son comportement, une stagiaire a mentionné que «[...] ce stage $\mathrm{m}^{\prime}$ avait permis de constater certaines forces et faiblesses $[\ldots]^{14}$. » Connaître ses forces et ses points à améliorer, c'est parfaire les premières et trouver des moyens de contrebalancer les deuxièmes. Le stage est l'occasion de se connaître davantage et de prendre conscience de son attitude en milieu de travail.

L'amélioration de certaines qualités est très présente en stage dont I'autonomie. À la DGDA, le fait d'avoir un horaire au jour le jour rassure l'étudiant puisqu'il voit davantage le travail à accomplir. Au fil des jours, il prend de l'assurance dans la réalisation de ses activités. Une fois qu'il a compris la tâche à accomplir et qu'il l'exécute bien, il devient efficace et performant. II peut refaire l'activité quelques jours plus tard sans aide et il gagne en autonomie. La confiance en soi est également citée à plusieurs reprises dans les travaux de stage des étudiants. Au début de leur stage, tous les stagiaires remettent en question leurs compétences et même leurs connaissances. Ils ont peur de faire des erreurs. Toutefois, la confiance en soi implique d'agir et de demander, s'il y a lieu, l'aide de son superviseur de stage. Les superviseurs de stage de la DGDA ne surveillent pas les stagiaires, ils leur font confiance et créent un climat propice à la discussion. Ils savent que les erreurs sont possibles en situation d'apprentissage. Pour améliorer sa confiance, le stagiaire doit les comprendre, les corriger et éviter qu'elles ne se reproduisent.

Pendant leur stage, les étudiants développent plusieurs autres qualités telles que la maturité, le sens des responsabilités, la capacité d'analyse, l'adaptation aux changements, l'efficacité, la curiosité, l'initiative et le développement de bonnes relations interpersonnelles. D'autres qualités sont directement liées aux activités de stage, par exemple hiérarchiser ses priorités, l'esprit d'équipe, l'ouverture face à la rétroaction, communiquer de façon professionnelle, oralement et par écrit. 


\subsection{Confirmer son choix professionnel}

Si les cours en archivistique donnent un aperçu de ce qu'est la profession, rien ne vaut une situation réelle pour nous aider à nous rendre compte de ce qui nous attend. En plus de confirmer notre domaine d'études, le stage permet de vérifier le choix et son aptitude à la profession. Le stagiaire connaît davantage ses préférences face aux tâches archivistiques, par exemple l'application de règles de conservation ou le traitement d'un fonds d'archives. II comprend aussi la dimension humaine de la profession, car elle est de plus en plus présente en archivistique. Cette dimension a un impact sur le choix des activités qu'il aimerait faire comme professionnel. Que ce soit de donner une formation à un groupe, rencontrer une unité administrative pour l'implantation d'une GED, animer une réunion ou gérer un conflit, le stagiaire se questionnera certainement sur ses capacités interpersonnelles et son intérêt pour ces activités.

Parmi les stagiaires de la DGDA, la très grande majorité d'entre eux sont demeurés dans le domaine archivistique après leur stage et ont obtenu des postes de techniciens en archivistique ou d'archivistes. Ils sont présents dans tous les secteurs (éducation, santé, entreprise privée, municipal, gouvernement). Suite à leur stage, certains finissants du certificat en archivistique ont poursuivi leurs études à la maîtrise en sciences de l'information. D'autres ont été engagés par la DGDA pour des contrats. Enfin, quelques-uns ont obtenu un poste à la Division et y travaillent encore aujourd'hui.

\subsection{Développer un réseau de contacts}

Mon stage à la Division de la gestion de documents et des archives a été déterminant dans mon court parcours archivistique, puisqu'il m'a permis d'apprendre sur plusieurs aspects de la profession, en plus de me bâtir un réseau de contacts. En effet, les stagiaires de la DGDA ont l'opportunité de recevoir une présentation de chacun des employés, qui expliquent la nature de leur travail ainsi que leur rôle au sein de l'équipe. J'ai beaucoup apprécié ces rencontres et la disponibilité du personnel à répondre à mes questions ${ }^{15}$.

Ces premiers échanges constituent le premier réseau de contacts du stagiaire. Une fois le stage terminé, le stagiaire peut recontacter ses 
anciens collègues s'il a un questionnement archivistique lors d'un emploi. Il peut aussi joindre son superviseur de stage s'il souhaite obtenir une référence pour un emploi.

\subsection{Devenir un professionnel}

Le stage est un outil de professionnalisation qui commence avant même le début de celui-ci. L'étudiant met à jour son curriculum vitæ, contacte son superviseur pour le rencontrer avant le stage afin de se présenter et de discuter des activités de stage. Par la suite, tout au long de son stage, l'étudiant apprend à devenir un professionnel par le développement et I'acquisition de compétences archivistiques et de qualités personnelles. II a une meilleure connaissance de soi, connaît ses ressources et ses limites, et améliore constamment sa manière de travailler. Le stagiaire a avantage à tirer profit des évaluations qu'il recevra de la part de son superviseur de stage. Les différentes rétroactions qu'il obtiendra l'aideront à progresser dans ses apprentissages tant personnels que professionnels. À la fin de son stage, l'étudiant a compris toutes les tâches liées à son stage. II sait aussi comment agir dans un milieu professionnel. Ces quelques semaines de stage lui permettent de s'identifier à la profession et de devenir un archiviste.

La fin du stage marque généralement la fin des études pour les étudiants et les prépare au marché du travail. L'expérience pratique du stage, particulièrement de la DGDA, est facilement redéployable dans d'autres milieux archivistiques. Cette première expérience en archivistique apporte une plus-value lors des entrevues d'embauche, car l'étudiant peut plus aisément «se vendre » auprès d'un employeur.

Les activités réalisées au moment du stage deviennent très utiles lors des emplois subséquents:

Le stage a finalement débouché sur plusieurs contrats dans ce même service et j'ai été appelé à participer à la mise en place de projets d'importance, incluant la numérisation sérielle et la gestion intégrée de documents. Ces expériences se sont avérées cruciales dans mon emploi actuel, alors qu'elles m'ont aidée à mener à bien les mandats de gestion et de diffusion numérique qui m'étaient confiés $[\ldots]^{16}$. 
J'ai maintenant un sens critique poussé quant aux procédures. J'ai créé plusieurs procédures ici sans que cela me soit demandé parce que je voyais le besoin. Mes procédures sont complètes et «idiot's proof »! !

\section{CONCLUSION}

Pour reprendre l'idée du philosophe Emmanuel Kant, la théorie sans la pratique est inutile, la pratique sans la théorie est aveugle (1905, p. 91). Le stage façonne le passage de la vie d'étudiant en archivistique (donc théorique) à la vie d'archiviste (plus pratique).

L'influence archivistique de la DGDA se transmet à ses stagiaires par les compétences acquises lors des activités professionnelles réalisées, par le fait d'être mieux outillés pour le marché du travail et par la passion qui anime les membres du personnel de la DGDA.

Ce qui a été le plus apprécié au-delà de l'acquis de connaissances et de contacts professionnels, ce fut le respect de l'individu dans un cadre académique. Les questions demeuraient toujours les bienvenues sans crainte de jugement de la part de mes évaluateurs. En somme, l'être humain s'est inscrit au cœur du travail archivistique, et la Division a réalisé beaucoup de ces objectifs avec discipline et un soupçon de convivialité. ${ }^{18}$

Pour terminer, je me sens privilégiée d'avoir réalisé mon stage au sein d'une équipe dynamique et soucieuse de la formation des archivistes de demain. II faut dire que ce fut un peu intimidant au début de se retrouver parmi les grands noms comme $\mathrm{M}^{\mathrm{me}}$ Baillargeon et $\mathrm{M}$. Champagne, mais ceux-ci (ainsi que tous les autres membres de l'équipe) ont été très ouverts et $\mathrm{m}^{\prime}$ ont accueillie comme une des leurs, ce qui fut apprécié et motivant ! ${ }^{19}$

Pour les étudiants, la DGDA est un modèle à suivre. Pour l'EBSI, elle représente aussi un modèle que nous donnons en exemple en classe et qui nous inspire lors de l'adaptation de nos programmes à l'évolution des pratiques archivistiques. Et pour le milieu professionnel, c'est aussi un modèle pour l'accueil et l'encadrement des étudiants, car elle sait 
mettre à profit la variété des profils des stagiaires et offre à ceux-ci des projets stimulants.

\section{NOTES}

1. Nous remercions vivement les anciens stagiaires de la DGDA qui ont fourni des commentaires sur leur stage.

2. Selon les descriptifs de l'Association des archivistes du Québec.

3. Cette partie s'inspire des réflexions des différentes coordonnatrices de stage qui se sont succédé à I'EBSI, particulièrement de Florence Arès.

4. Note de la rédaction: Michel Champagne a écrit un article intitulé Les archives définitives à la DGDA: accroissement, traitement, diffusion publié dans ce numéro.

5. Entre autres, le Fonds de l'Association des femmes diplômées des universités, le Fonds Auguste Descarries, le fonds de l'Association des diplômés et diplômées en théologie de l'Université de Montréal ont été traités par des stagiaires.

6. Par exemple, le Fonds Léo Lortie, le Fonds Paul Loyonnet, le Fonds Guillaume Couture, le Fonds Peter Jacobs ont été traités par des stagiaires.

7. Siham Belghaitar

8. Ana-Maria Drobota

9. Ana-Maria Drobota

10. Caroline Duclos

11. Sandie Gilbert

12. Olivier Dumas

13. Marie-France Vaillancourt

14. Caroline Gadoury

15. Noura Elmobayeb-Langevin

16. Nicolas Bednarz

17. Sylvie Aubin

18. Olivier Dumas

19. Noura Elmobayed-Langevin 


\section{BIBLIOGRAPHIE}

ARÈS, F. (1989). Le stage et la formation universitaire. Archives, 20(3), 53-60.

CHAMPAGNE, M. (2010-2011). Le rôle stratégique du stage dans la formation en archivistique: réflexions d'un superviseur de stages. Archives, 42(2), 107-116.

ÉCOLE DE BIBLIOTHÉCONOMIE ET DES SCIENCES DE L'INFORMATION (EBSI). (2015). Guide de stage. Maîtrise en sciences de l'information, orientation professionnelle. Montréal, Québec: Université de Montréal.

KANT, E. (1905). Critique de la raison pure. Paris: Félix Alcan éditeur.

VILLENEUVE, L. (1995). Cahier d'encadrement du stage supervisé. Montréal, Québec: Éditions Saint-Martin.

VILLENEUVE, L. (1994). L'encadrement du stage supervisé. Montréal, Québec: Éditions Saint-Martin. 